\title{
Global survey of miRNAs and tRNA-derived small RNAs from the human parasitic protist Trichomonas vaginalis
}

Zhen-Sheng Wang ${ }^{1,2}$, Hong-Chang Zhou ${ }^{3}$, Chun-Yan Wei ${ }^{1}$, Zhi-Hua Wang ${ }^{4}$, Xiao Hao ${ }^{5}$, Lian-Hui Zhang ${ }^{1}$, Jing-Zhong Li ${ }^{6}$, Zeng-Lei Wang ${ }^{2^{*}}$ and Heng Wang ${ }^{1 *}$

\begin{abstract}
Background: Small non-coding RNAs play critical regulatory roles in post-transcription. However, their characteristics in Trichomonas vaginalis, the causative agent of human sexually transmitted trichomoniasis, still remain to be determined.

Methods: Small RNA transcriptomes from Trichomonas trophozoites were deep sequenced using the Illumina NextSeq 500 system and comprehensively analyzed to identify Trichomonas microRNAs (miRNAs) and transfer RNA (tRNA)derived small RNAs (tsRNAs). The tsRNA candidates were confirmed by stem-loop quantitative reverse transcription$P C R$, and motifs to guide the cleavage of tsRNAs were predicted using the GLAM2 algorithm.

Results: The miRNAs were found to be present in T. vaginalis but at an extremely low abundance $(0.0046 \%)$. Three categories of endogenous Trichomonas tsRNAs were identified, namely 5 'tritsRNAs, mid-tritsRNAs and 3'tritsRNAs, with the $5^{\prime}$ tritsRNAs constituting the dominant category (67.63\%) of tsRNAs. Interestingly, the cleavage site analysis verified both conventional classes of tRNA-derived fragments (tRFs) and tRNA-halves in tritsRNAs, indicating the expression of tRNA-halves in the non-stress condition. A total of 25 tritsRNAs were experimentally confirmed, accounting for $78.1 \%$ of all tested candidates. Three motifs were predicted to guide the production of tritsRNAs. The results prove the expression of tRFs and tRNA-halves in the T. vaginalis transcriptome.

Conclusions: This is the first report of genome-wide investigation of small RNAs, particularly tsRNAs and miRNAs, from Trichomonas parasites. Our findings demonstrate the expression profile of tsRNAs in T. vaginalis, while miRNA was barely detected. These results may promote further research aimed at gaining a better understanding of the evolution of small non-coding RNA in T. vaginalis and their functions in the pathogenesis of trichomoniasis.
\end{abstract}

Keywords: Trichomonas vaginalis, Transfer RNA, tRNA-derived small RNAs, tRFs, tRNA-halves, Trichomoniasis

\footnotetext{
*Correspondence: zengleiwang@pumc.edu.cn; wangh@ibms.cams.cn

${ }^{1}$ Department of Microbiology and Parasitology, Institute of Basic

Medical Sciences, Chinese Academy of Medical Sciences, School

of Basic Medicine, Peking Union Medical College, \#5 Dong Dan San Tiao,

Beijing 100005, People's Republic of China

${ }^{2} \mathrm{NHC}$ Key Laboratory of Systems Biology of Pathogens, Institute

of Pathogen Biology, Chinese Academy of Medical Sciences and Peking

Union Medical College, Beijing, People's Republic of China

Full list of author information is available at the end of the article
}

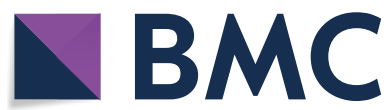

c The Author(s) 2021. This article is licensed under a Creative Commons Attribution 4.0 International License, which permits use, sharing, adaptation, distribution and reproduction in any medium or format, as long as you give appropriate credit to the original author(s) and the source, provide a link to the Creative Commons licence, and indicate if changes were made. The images or other third party material in this article are included in the article's Creative Commons licence, unless indicated otherwise in a credit line to the material. If material is not included in the article's Creative Commons licence and your intended use is not permitted by statutory regulation or exceeds the permitted use, you will need to obtain permission directly from the copyright holder. To view a copy of this licence, visit http://creativeco mmons.org/licenses/by/4.0/. The Creative Commons Public Domain Dedication waiver (http://creativecommons.org/publicdomain/ zero/1.0/) applies to the data made available in this article, unless otherwise stated in a credit line to the data. 


\section{Background}

Small non-coding RNAs (snRNAs) have emerged as essential post-transcriptional regulators in a myriad of cellular and organismal processes and have consequently become an important focus of research in the past three decades. Since the discovery of microRNAs (miRNAs) in 1993 [1], the repertoire of snRNAs has greatly expanded through continuous characterization of new small RNAs from various origins, facilitated by the advance of deep sequencing technologies. In addition to such well-established snRNAs as miRNAs, endogenous small interference RNAs (endosiRNAs) and P-element induced wimpy testis (PIWI) protein-interacting RNAs (piRNAs), transfer RNA (tRNA)-derived snRNAs have remained a highly studied topic in recent years, and their important roles in a wide variety of biological and pathological situations have been increasingly recognized.

tRNA-derived small RNAs (tsRNAs) can be broadly categorized into two main classes that are likely dependent on their biogenesis: tRNA-halves and tRNA-derived fragments (tRFs). The conventional tRNA-halves are produced by a single ribonucleolytic cleavage within the anticodon loops of mature tRNAs under stress conditions, and thus they have also been referred to as tRNA-derived stress-induced small RNAs (tiRNAs) [2-6]. However, a recent study presented evidence of tRNA-halves being expressed in a non-stress condition [7], suggesting that the factors affecting the genesis of tRNA-halves might need to be re-evaluated. Endonucleolytic cleavages of both mature and precursor tRNAs near the D-,anticodon-,or T $\psi \mathrm{C}$-arm lead to the generation of tRFs. Despite the need for a consistent nomenclature to describe the types of tRFs, they are typically classified into four subclasses: $5^{\prime} \mathrm{tRFs}, 3^{\prime} \mathrm{tRFs}$ (or $3^{\prime} \mathrm{CCA}$ tRFs), $3^{\prime} \mathrm{UtRFs}$ (tRF-1) and internal tRFs (itRFs or mid-tRFs) in terms of their positions mapped to tRNAs [8-10]. tRFs are constitutively and conservatively expressed in organisms from lower archaea to mammals; however, their biological roles and mechanisms of action remain largely unclear.

Research to date has associated tsRNAs with diverse human diseases and infections, revealing functions in the regulation of cell apoptosis, cell viability, RNA degradation, RNA stability, translational repression and cellular proliferation $[6,11-14]$. Most interestingly, tRFs have the ability to behave like canonical miRNAs and regulate target gene expression through essential interactions with the Argonaute (AGO) family [15, 16], PIWI [17] or DICER proteins [18]. The similarity of tRFs and miRNAs has also resulted in misannotation and cross-mapping of these two groups. In some species that lack miRNA molecules, such as Plasmodium parasites, tRFs might act as alternative regulators to accomplish the intense post-transcriptional regulation needed during the rapid morphological change undergone by these organisms [10]. In the flagellated protozoan Giardia lamblia, the abundant tRFs detected were involved in the differentiation process, while miRNAs that had been previously reported were found to be absent or misannotated [19]. tRFs have also been discovered in other protozoan parasites, including Tetrahymena and Trypanosoma cruzi [20], as well as from exosomes of Leishmania donovani [21], implying their conservative expression in primitive eukaryotes, although their functions need to be further investigated.

Trichomonas vaginalis is an extracellular, unicellular flagellated protozoan parasite that belongs to the same class of Zoomastigophorea as G. lamblia. Infection of $T$. vaginalis leads to the occurrence of trichomoniasis, which remains the most prevalent non-viral sexually transmitted disease in humans, affecting 142.6 million people annually [22]. Trichomonas vaginalis parasites undergo dramatic biological changes even within the single trophozoite stage, including morphological change, DNA replication, multiple nucleus divisions, transposon activities and lateral gene transfer during differentiation. It is possible that the parasites take the advantage of certain snRNAs, such as tRNA-halves, tRFs or miRNAs, in these complicated changes. To date, miRNAs have only been reported by in silico prediction or hairpin-loop searching in T. vaginalis [23-25]. However, the misinterpretation of miRNAs discovered using the same methods in a closely related parasite, G. lamblia [19], hint at an ambiguous fate of Trichomonas miRNA molecules. Albeit a recent study revealed the occurrence of nine tRNA-halves in extracellular vesicles from $T$. vaginalis [7], their global expression profile in this parasite still remains to be revealed. It is therefore necessary to revisit the small RNAs in T. vaginalis, in particular tsRNAs and miRNAs, using advanced high-throughput deep sequencing to achieve a better understanding of the snRNAs in this parasite.

In the study reported here, we deeply sequenced the small RNA transcriptome from Trichomonas trophozoites. Based on our results from a genome-wide comprehensive analysis and experimental verification, we report for the first time the global identification and characterization of endogenous Trichomonas tsRNAs that may play a pivotal role in parasite development. In contrast, analysis of our sequencing data barely detected miRNAs.

\section{Methods}

T. vaginalis maintenance and RNA isolation

Three original clones (Tv01, Tv02 and Tv03) of T. vaginalis strains P7, which was isolated and cloned in 2012 
and stored in liquid nitrogen until use, were maintained in Diamond's media supplemented with $10 \%$ heatinactivated bovine serum, penicillin $(100$ units $/ \mathrm{ml})$ and streptomycin $(0.1 \mathrm{mg} / \mathrm{ml})$ at $37^{\circ} \mathrm{C}$ without extra iron, as previously described [26]. The morphology of the parasites was checked daily to ensure the maintenance of typical pear-shaped trophozoites during culture. Parasite viability and density were monitored by Trypan blue exclusion on the hemocytometer. Fewer than $1 \times 10^{6}$ trophozoites/ml was maintained during culture to avoid overgrowth. The pellet of each isolate was collected at the exponential phase of the trophozoite by centrifugation. Total RNA was extracted employing TRIzol Reagent (Invitrogen, Shanghai, China) and treated with DNase I to remove any genomic DNA contamination. RNA was then separated by urea-denatured $15 \%$ polyacrylamide gel electrophoresis, and bands of small RNAs with a length of 12-40 nucleotides (nt) were extracted and purified for further use.

\section{Small RNA library construction and deep sequencing}

To avoid the interference of numerous post-transcriptional modifications in tRNA during complementary DNA (cDNA) synthesis and adapter ligation in RNAsequencing, the following treatments were completed before library construction: (i) both $3^{\prime}$-aminoacyl deacylation and $2^{\prime}, 3^{\prime}$-cyclic phosphate removal to $3^{\prime}-\mathrm{OH}$ for $3^{\prime}$ adaptor ligation; (ii) $5^{\prime}$-hydroxyl group phosphorylation to $5^{\prime}$-phosphorylation for $5^{\prime}$-adaptor ligation; (iii) $\mathrm{m} 1 \mathrm{~A}$ and $\mathrm{m} 3 \mathrm{C}$ demethylation for efficient reverse transcription. The construction and subsequent sequencing of small RNA libraries were accomplished following commercial protocols. Briefly, small RNA molecules were ligated to $5^{\prime}$ and $3^{\prime}$ adaptors consecutively and then converted to cDNA by reverse transcription followed by PCR amplifications (RT-PCR). Approximately $2.34 \mathrm{fmol}$ of RT-PCR products per sample were sequenced directly by the Illumina NextSeq 500 system (Illumina Inc. San Diego, CA, USA) at 50 bp single-read by Aksomics Inc. (Shanghai, China).

\section{Small RNA analysis and tsRNA identification}

The quality of raw sequencing data was evaluated using FastQC (v0.11.7). The $3^{\prime}$ and $5^{\prime}$ adaptors were trimmed and reads shorter than $18 \mathrm{nt}$ or longer than $40 \mathrm{nt}$ were filtered out by Cutadapt (v1.17) and Python2 (v2.7.5) to remove any contamination and yield clean data. The clean dataset was further mapped to the genome of $T$. vaginalis G3 strain (TrichDB, release 47; http://trich db.org/trichdb/) using BLASTN. The clean-read counts were normalized as a relative number per one million reads (RPM) and analyzed in both the total and unique read categories to indicate the abundance and diversity of reads, respectively. To investigate tRNA-derived segments, the data were further aligned to a total of $165 \mathrm{~T}$. vaginalis tRNA genes downloaded from the TrichDB database (http://trichdb.org/trichdb/) by performing BLASTN alignment. The genome-wide expression intensity of the T. vaginalis tsRNAs was sequentially calculated with the "Build" function of Bowtie2 (v2.1.0.0) and the "coverageBed" function of Bedtools (v2.29.2). Data extraction of each type of tsRNAs was performed by compiled Perl (v5.22) codes. The statistical analysis for the Pearson correlation coefficients was carried out utilizing the "cor" function in R package stats. The ggplot2 package (v3.3.1) in R language (v3.5.0) was employed to create all the plots.

\section{Motif analysis}

The motifs at the cleavage sites of tritsRNAs were predicted by using the GLAM2 algorithm in Gapped Local Alignment of Motifs (GLAM2 v1056) [22] with default parameters adopted, except that only the given strand was aligned.

\section{Stem-loop RT-PCR}

The templates of total RNAs were reverse-transcribed into cDNAs utilizing the Goldenstar ${ }^{\mathrm{TM}}$ RT6 cDNA Synthesis Kit (Beijing TsingKe Biotech Co. Ltd., Beijing, China) following the manufacturer's instructions. Specific stem-loop reverse transcription primers (Additional file 1: Table S1) were designed in accordance with the sequence of each tritsRNA candidate, as described previously $[27,28]$. The PCR was carried out with an initial denaturation at $98{ }^{\circ} \mathrm{C}, 2 \mathrm{~min}$; followed by denaturation at $98{ }^{\circ} \mathrm{C} / 10 \mathrm{~s}$, annealing at $55{ }^{\circ} \mathrm{C} / 10 \mathrm{~s}$ and extension at $72{ }^{\circ} \mathrm{C} / 15 \mathrm{~s}$, for 35 cycles; with a final extension at $72{ }^{\circ} \mathrm{C}$ for $5 \mathrm{~min}$. The PCR products were evaluated in a $12 \%$ polyacrylamide gel. The expected sizes of the PCR products were estimated by the length of each tsRNAs, with additional $\sim 40 \mathrm{bp}$ of nucleotides technically introduced into the stem-loop primers. Negative controls lacking DNase I-treated RNA or reverse transcriptase in the RT reactions or template in the PCR analyses were applied to validate the accuracy and specificity of the stem-loop RTPCR analyses. The PCR primers are listed in Additional file 1: Table S2.

\section{Results}

\section{Absence of miRNAs in T. vaginalis}

Three $T$. vaginalis strains were employed for deep sequencing of the 18- to 40-nt small RNAs. As shown in Table 1, the three libraries yielded 6,303,530, 7,071,840 and 5,809,794 reads, respectively, that perfectly mapped to the $T$. vaginalis genome, accounting for an average of $91.76 \%$ of the clean data. The distribution of mapped 
reads from these three isolates was relatively consistent, all displaying three main peaks at 29, 32 and $36 \mathrm{nt}$ (Fig. 1a). Consequently, these data were pooled together for further analysis to enhance the identification of any novel type of small RNAs. Apart from these three major peaks, in total reads, a single peak at 34 nt showed in unique reads (Fig. 1b). This feature was incredibly different from those of model organisms, which generally have demonstrated a peak at $\sim 22 \mathrm{nt}$ dominated by miRNAs in both the total and unique reads [29-31] and suggests that the composition of small RNAs in T. vaginalis might be divergent.

The data were then annotated, and the abundance of each type of small RNA was assessed in terms of its expression level. As illustrated in Fig. 1c, these small RNAs originated from ribosomal RNAs (rRNAs), tRNAs, messenger RNAs (mRNAs), small nuclear RNAs, small nucleolar RNAs (snoRNAs), as well as unannotated small RNAs. Unexpectedly, there was an extremely low abundance of miRNAs in our pool $(0.0046 \%)$. Among the 27 miRNAs identified previously [23-25], only nine were detected in our deep sequencing data, and these had particular low counts, with the exception of 'tvm-005' derived from tRNA (Additional file 1: Table S3). In contrast, tsRNAs predominated after the rRNA-derived reads and accounted for $12.79 \%$ of clean reads. These molecules prevailed in $T$. vaginalis genomic contig DS113177 to DS127907, from where tRNA genes were mainly coded (Fig. 1d).

\section{Profiles of tsRNAs from $T$. vaginalis}

To explore the expression profiles of tsRNAs, we analyzed their 20-amino acid parental tRNAs. Eight amino acids, namely Glu, Gly, Phe, Lys, Val, Arg, Asn and Tyr, were found to comprise up to $85 \%$ of all tsRNAs (Fig. 2a). This expression bias indicated that the tsRNAs were not generated by the random degradation of mature tRNAs. The distribution pattern of tsRNAs was investigated further and in addition to the distribution shown in Fig. 1b, there were only two conspicuous peaks, at $\sim 29$ and $\sim 33$ nt, that dominated in the total tsRNAs (Fig. 2b), implying multiple types of tsRNAs with diverse sizes in T. vaginalis. These tsRNA reads were therefore mapped to all 165 Trichomonas tRNAs. As expected, their biogenesis from the parental tRNAs was found to be relatively conserved, as previously reported $[10,19-21]$, with a large number of reads aligned to three main positions at the $5^{\prime}$ end, anticodon area and $3^{\prime}$ end of mature tRNAs (Fig. 2c). Consequently, these three types of Trichomonas tsRNAs were named $5^{\prime}$ tritsRNAs, mid-tritsRNAs and $3^{\prime}$ tritsRNAs, respectively. The homogeneity of these tritsRNAs based on their types and size distributions were investigated by plotting the RPM values against sizes in both
Table 1. Mapping and classification of reads against the Trichomonas vaginalis genome

\begin{tabular}{lllll}
\hline Reads & Clone Tv01 & Clone Tv02 & Clone Tv03 & Pooled data \\
\hline Clean reads & $6,909,748$ & $7,811,640$ & $6,186,244$ & $20,907,632$ \\
Un-mapped reads & 606,218 & 739,800 & 375,450 & $1,721,468$ \\
Mapped reads & $6,303,530$ & $7,071,840$ & $5,809,794$ & $19,185,164$ \\
mRNAs & 190,790 & 322,082 & 651,191 & $1,164,063$ \\
5'UTR & 3765 & 5245 & 7938 & 16,948 \\
CDS & 186,694 & 316,213 & 642,807 & $1,145,714$ \\
3'UTR & 1219 & 1648 & 2095 & 4962 \\
rRNAs & $2,772,196$ & $2,639,586$ & $1,807,762$ & $7,219,544$ \\
tRNAs & 955,479 & $1,076,990$ & 624,938 & $2,657,407$ \\
miRNAs & 39,596 & 57,388 & 44,879 & 141,863 \\
sn/snoRNAs & 1331 & 1521 & 1532 & 4384 \\
Unannoted small & $2,344,138$ & $2,974,273$ & $2,679,492$ & $7,997,903$ \\
RNAs & & & & \\
\hline
\end{tabular}

CDS, Coding sequence; $\mathrm{m} / \mathrm{mi} / \mathrm{r} / \mathrm{tRNA}$, messenger/micro/ribosomal/transfer RNA, respectively; sn/snoRNA, small nuclear/nucleolar RNA, respectively; UTR, untranslated region

the total and unique reads. Two peaks were observed for 5 'tritsRNAs, at $\sim 29 \mathrm{nt}$ and $\sim 33 \mathrm{nt}$; one peak was observed for mid-tritsRNAs, at $\sim 21 / 22 \mathrm{nt}$; and two peaks were observed for 3 'tritsRNAs, at $\sim 24$ nt and $\sim 40$ nt, respectively (Fig. 2d), displaying a great difference in their sizes. The double peaks that occurred in the plots of $5^{\prime}$ tritsRNAs and $3^{\prime}$ tritsRNAs suggested that there were at least two subgroups of tritsRNAs in each type.

We subsequently selected the top 20 tRNAs that produced high levels of tritsRNAs to further evaluate their expression divergence. Among these, seven primarily produced $5^{\prime}$ tritsRNAs, three generated mid-tritsRNAs and five created 3 tritsRNAs; the remaining yielded tritsRNAs from multiple regions (Fig. 2c). 5 'tritsRNAs were the dominant type (67.62\%) of tritsRNAs, with mid-tritsRNAs and 3 'tritsRNAs accounting for only $7.37 \%$ and $15.86 \%$, respectively (Fig. 2e). No significant association between the abundance of any two types of tritsRNAs was found by Pearson correlation analysis (Additional file 2: Figure S1). The predominating tritsRNAs in each group were then examined by plotting the RPM values of all 60 tritsRNAs from these tRNAs against their sizes. A total of 32 tritsRNAs were identified to show consistent peaks, as shown in Fig. 2d, including nine 5 'tritsRNAs, 12 mid-tritsRNAs and $113^{\prime}$ tritsRNAs, as shown in Additional file 3: Figure S2, Additional file 4: Figure S3, Additional file 5: Figure S4 and in Table 2. These 32 tritsRNAs were then used for further classification and confirmation analysis.

\section{Identification of tritRFs and tritRNA-halves}

tRFs, which are typically shorter than 32 nt [8], are the theoretical products of tsRNAs under ordinary 


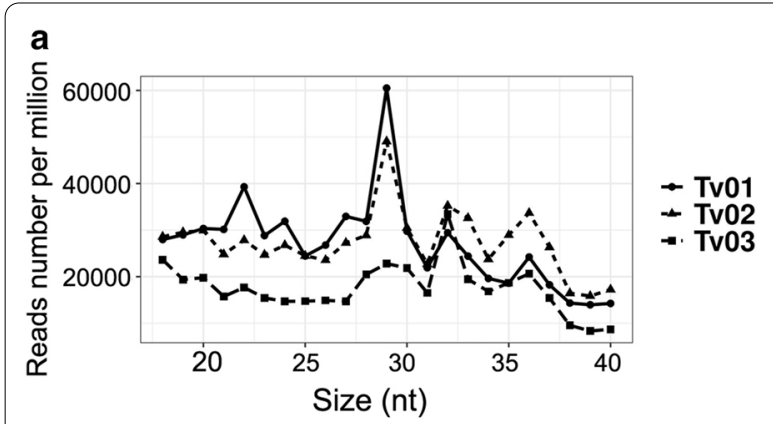

C

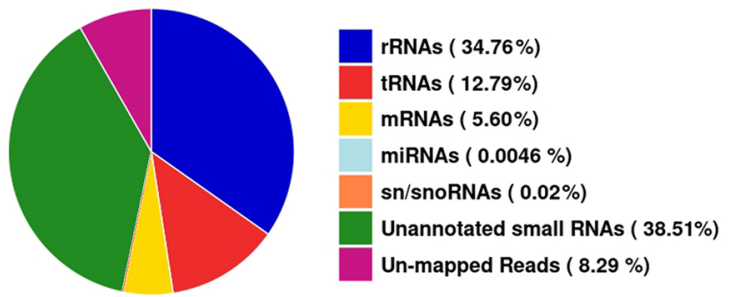

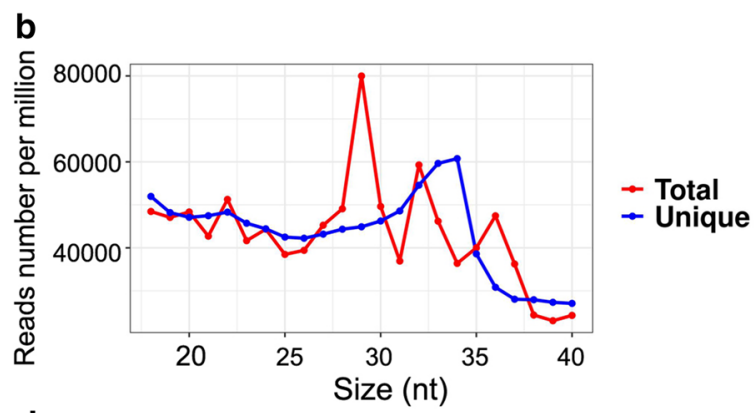

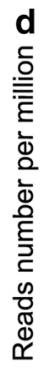

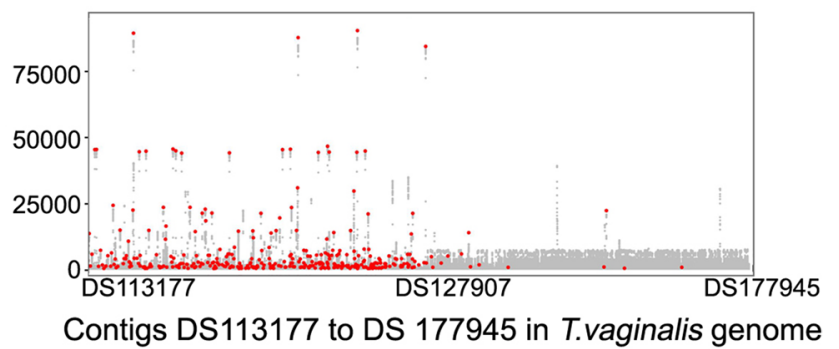

Fig. 1. General features of 18- to 40-nt-long small RNAs from Trichomonas vaginalis. a, b Size distributions of small RNAs from individual samples (a) and pooled data (b). c Abundance of small RNA populations with reference to the T. vaginalis genomic annotation. $\mathbf{d}$ Genome-wide density analysis of small RNAs along all contigs of T. vaginalis genome. Red dots represent tsRNAs. tsRNAs Transfer RNA-derived small RNAs

conditions; however, our analysis identified longer products in both $3^{\prime}$ and $5^{\prime}$ tritsRNAs. To further classify these tritsRNAs, we investigated their cleavage sites by aligning each of those 32 tritsRNAs to its parental tRNA. As illustrated in Fig. 3, two sites were identified that produced $5^{\prime}$ tritsRNAs, with one occurring in the anticodon loop, generating the 33-nt $5^{\prime}$ tritsRNAs, and one in the anticodon arm, yielding the 29-nt $5^{\prime}$ tritsRNAs. The mid-tritsRNAs originated from two sets of combined endonucleolytic cleavages, both of which occurred in the arms neighboring the anticodon loop and $\mathrm{T} \psi \mathrm{C}$ loop; however, one group covered the anticodon loop while the other involved the $\mathrm{T} \psi \mathrm{C}$ loop. Three cleavage sites in anticodon loop, anticodon arm and $\mathrm{T} \psi \mathrm{C}$ arm resulted in the generation of $3^{\prime}$ tritsRNAs with sizes of 40, 33 and $24 \mathrm{nt}$, respectively. Therefore, 29-nt $5^{\prime}$ tritsRNAs, mid-tritsRNAs and 24- and 33-nt 3'tritsRNAs were produced by combined cleavages other than that in the anticodon loop and belonged to the class of tritRFs, while two types (33-nt $5^{\prime}$ tritsRNAs and 40-nt $3^{\prime}$ tritRNAs) were generated by a single cleavage in the anticodon loop and thus were classified as tritRNAhalves (Table 2).

\section{Experimental confirmation of tritsRNA candidates and motif prediction}

We further experimentally evaluated the presence of tritRFs and tritRNA-halves in $T$. vaginalis to confirm our findings. Stem-loop RT-PCR successfully amplified 25 (9 $5^{\prime}$ tritsRNAs, 7 mid-tritsRNAs, $93^{\prime}$ tritsRNAs) of these 32 candidates, corresponding to $78.1 \%$ of the candidates tested. This result proved that both tritRFs and tritRNAhalves were expressed in the $T$. vaginalis transcriptome (Fig. 4a).

The motifs around the cleavage sites that might guide the generation of tritsRNAs were then explored. Employing the GLAM2 algorithm, we successfully predicted that three motifs (Motifs TV1-3; Fig. 4b) would process tritsRNAs. Motif TV1 was located along the D-stem and anticodon-arm for the $3^{\prime}$ end cleavage of $5^{\prime}$ tritsRNAs (29 nt). Motif TV2 and TV3 were both in the $\mathrm{T} \psi \mathrm{C}$ stem to process the $3^{\prime}$ end cleavage of midtritsRNAs (22 nt) and $5^{\prime}$ end cleavage of $3^{\prime}$ tritsRNAs (24 nt), respectively. Albeit both motif TV2 and TV3 contained the highly conservative 'UUC' triple-nucleotide element, they seemed to be controlling the generation of various types of tritsRNAs (Fig. 4b).

\section{Discussion}

Despite the critical regulatory roles of snRNAs in posttranscription, our knowledge of their characteristics, biogenesis and functions in lower eukaryotes remains limited. The recent rapid development of high-throughput sequencing technology has greatly advanced research on small non-coding RNAs, revealing a variety of small RNAs, including those formerly misclassified tsRNAs. 


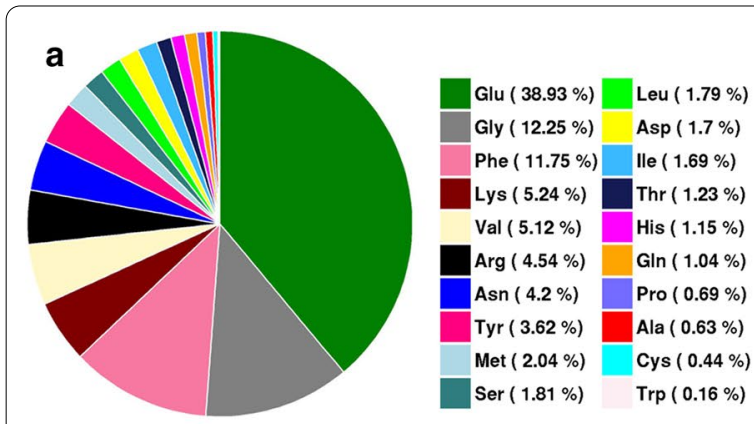

b

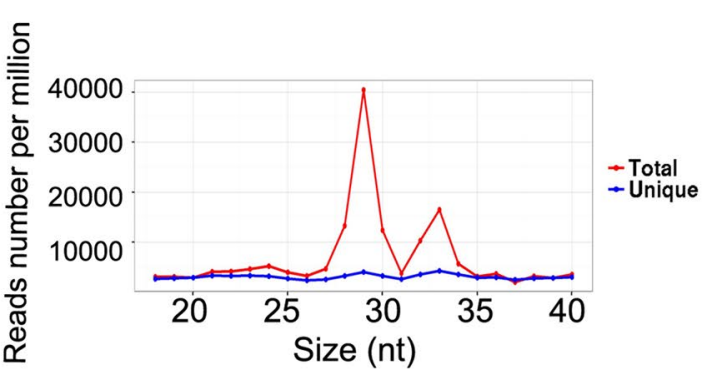

C
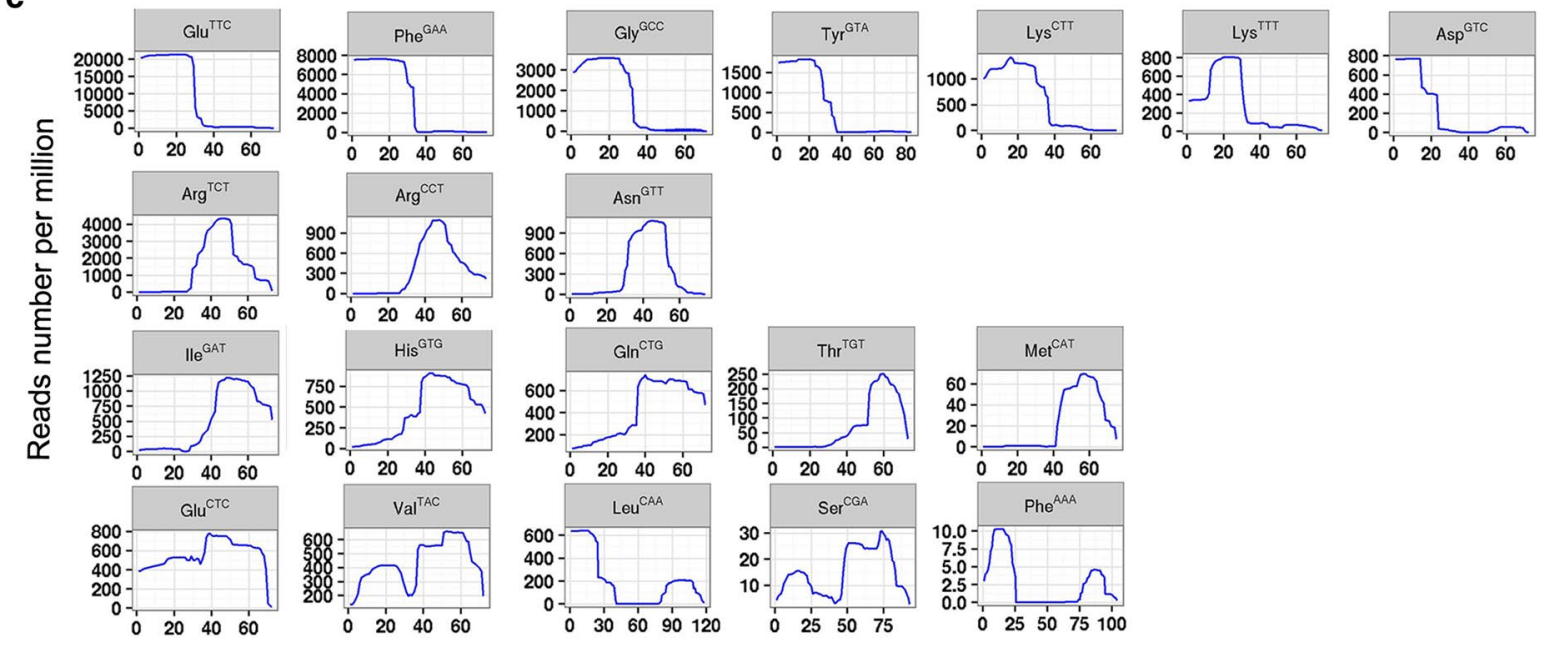

Size (nt)
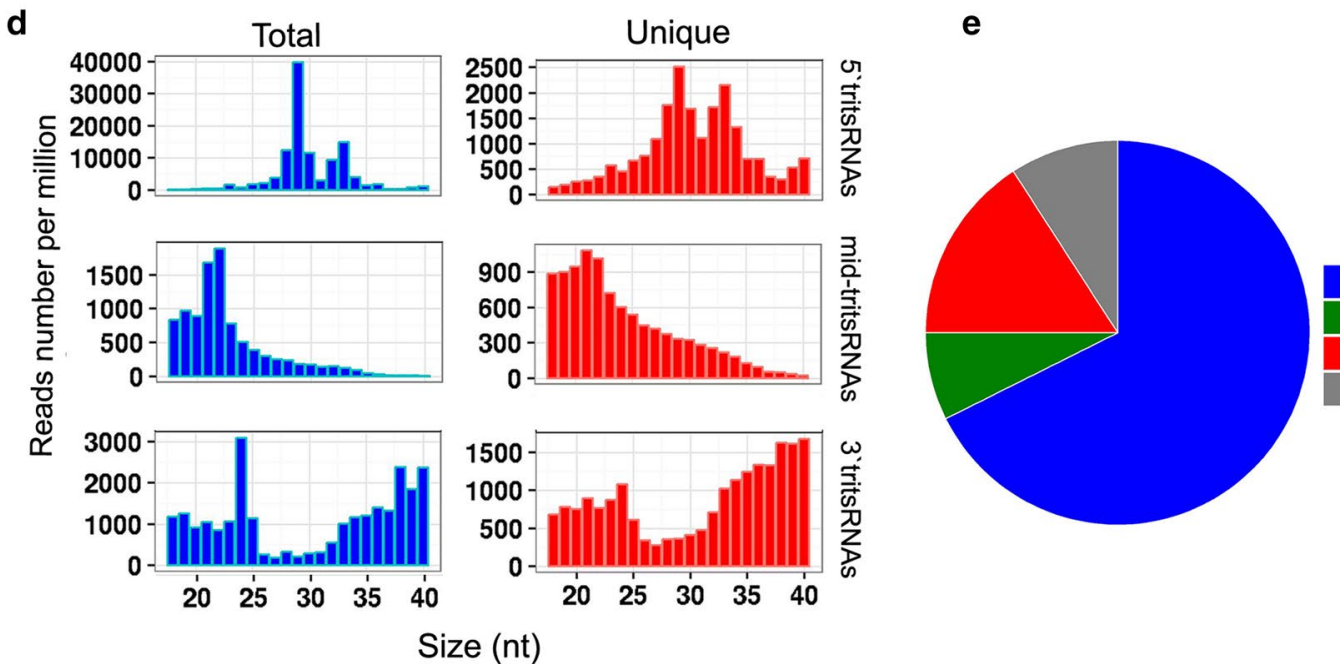

$5 `$ tsRNAs $(67.63 \%)$ mid-tsRNAs ( $7.38 \%$ ) $3 `$ tsRNAs $(15.86 \%)$ The rest $(9.13 \%)$

Fig. 2. Profiles of tRNA-derived small RNAs from T. vaginalis. a Frequency of tsRNAs originating from tRNAs, $\mathbf{b}$ size distributions of tsRNAs, $\mathbf{c}$ coverage range analysis of tritsRNAs among the top 20 highest tsRNA-expressed tRNAs, $\mathbf{d}$ size distributions of three types of tritsRNAs in both the total and unique reads, e frequency of three categories of tsRNAs 
Table 2. Classification of tritsRNA candidates

\begin{tabular}{|c|c|c|c|}
\hline Category & Class & Parental tRNAs & Size (nt) \\
\hline \multirow[t]{2}{*}{$5^{\prime}$ tritsRNAs } & $5^{\prime}$ tritRFs & 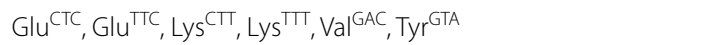 & 29 \\
\hline & $5^{\prime}$ tritRNA-halves & $P h e^{G A A}, G l y^{G C C}, V_{a l} l^{T A C}$ & 33 \\
\hline mid-tritsRNAs & mid-tritRFs & 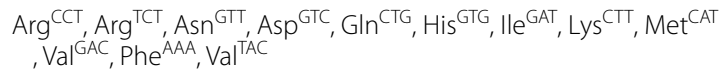 & $21 / 22$ \\
\hline \multirow[t]{3}{*}{ 3'tritsRNAs } & $3^{\prime}$ tritRFs & $\mathrm{Glu}^{T \mathrm{TC}}, \mathrm{Val}{ }^{\mathrm{TAC}}, \mathrm{Lys}^{\mathrm{CTT}}$ & 24 \\
\hline & $3^{\prime}$ tritRFs & $\operatorname{Arg}^{\mathrm{CCT}}, \mathrm{Arg}^{\mathrm{TCT}}, \mathrm{Glu} \mathrm{u}^{\mathrm{CTC}}$ & 33 \\
\hline & $3^{\prime}$ tritRNA-halves & Leu $^{\mathrm{CAA}}, \mathrm{Val}^{\mathrm{GAC}}, \mathrm{Thr}^{\mathrm{TGT}}, \| \mathrm{e}^{\mathrm{GAT}}, \mathrm{Met}^{\mathrm{CAT}}$ & 40 \\
\hline
\end{tabular}

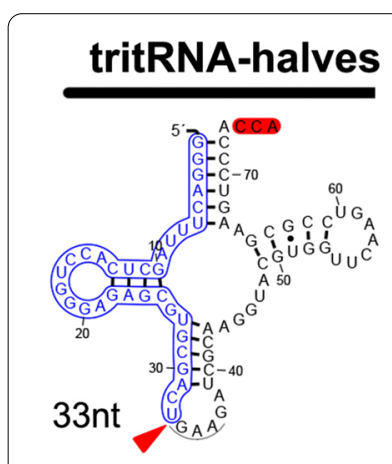

\section{5'tsRNA of Phe GAA $^{\text {ta }}$}

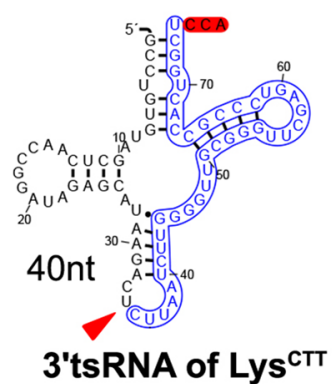

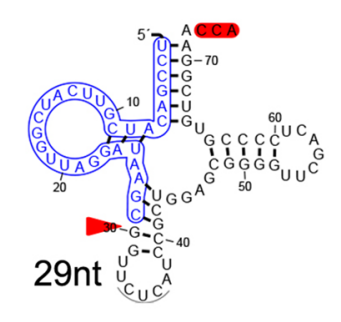

5'tsRNA of GlucTc

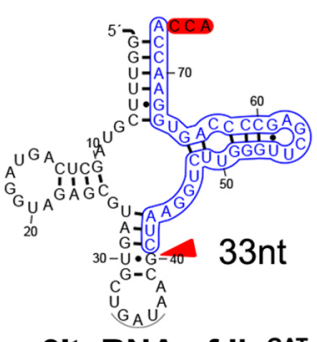

3'tsRNA of Ile

\section{tritRFs}

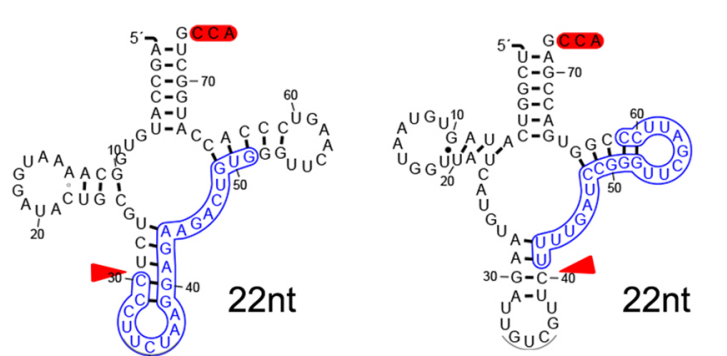

mid-tsRNA of Arg $^{\text {TCT }}$

mid-tsRNA of Asp ${ }^{\mathrm{GTC}}$

Fig. 3. Cleavage sites of tritRNA-halves and of tritRFs in schematic mature tRNAs. The tritsRNA sequences are highlighted in blue, and cleavage sites are indicated by red triangles. RF RNA-derived fragments

In this study, we used deep sequencing techniques and genome-wide studies to investigate small RNAs from the human parasite $T$. vaginalis. Strikingly, miRNAs occurred at a particularly low abundance $(0.0046 \%)$ in our dataset. Although previous studies have reported 27 miRNAs based on in silico prediction or hairpinloop searching, only nine were identified in our analysis. Two of them were derived from tRNAs and classified as $5^{\prime}$ tritsRNA-Gln $^{\text {TTG }}$ and $5^{\prime}$ tritsRNA-Thr $^{\text {TGT }}$, and one was apparently generated from $16 \mathrm{~S}$ ribosomal RNA [23]. The extremely low abundance of miRNAs may indicate that their biological role is minimal or that they are not even present in $T$. vaginalis. It has been shown that miRNA is either absent or has no biological function in protists such as Plasmodium falciparum [10], Giardia lamblia [19] and Leishmania parasites [32], suggesting that these parasites likely employ other molecules to complete gene regulation. For example, tsRNAs have been reported to be involved in Giardia lamblia parasite differentiation [19]. In mammalian cells, tsRNAs have also been found to participate in translational regulation by binding a multienzyme complex [33], ribosomes [34] and 

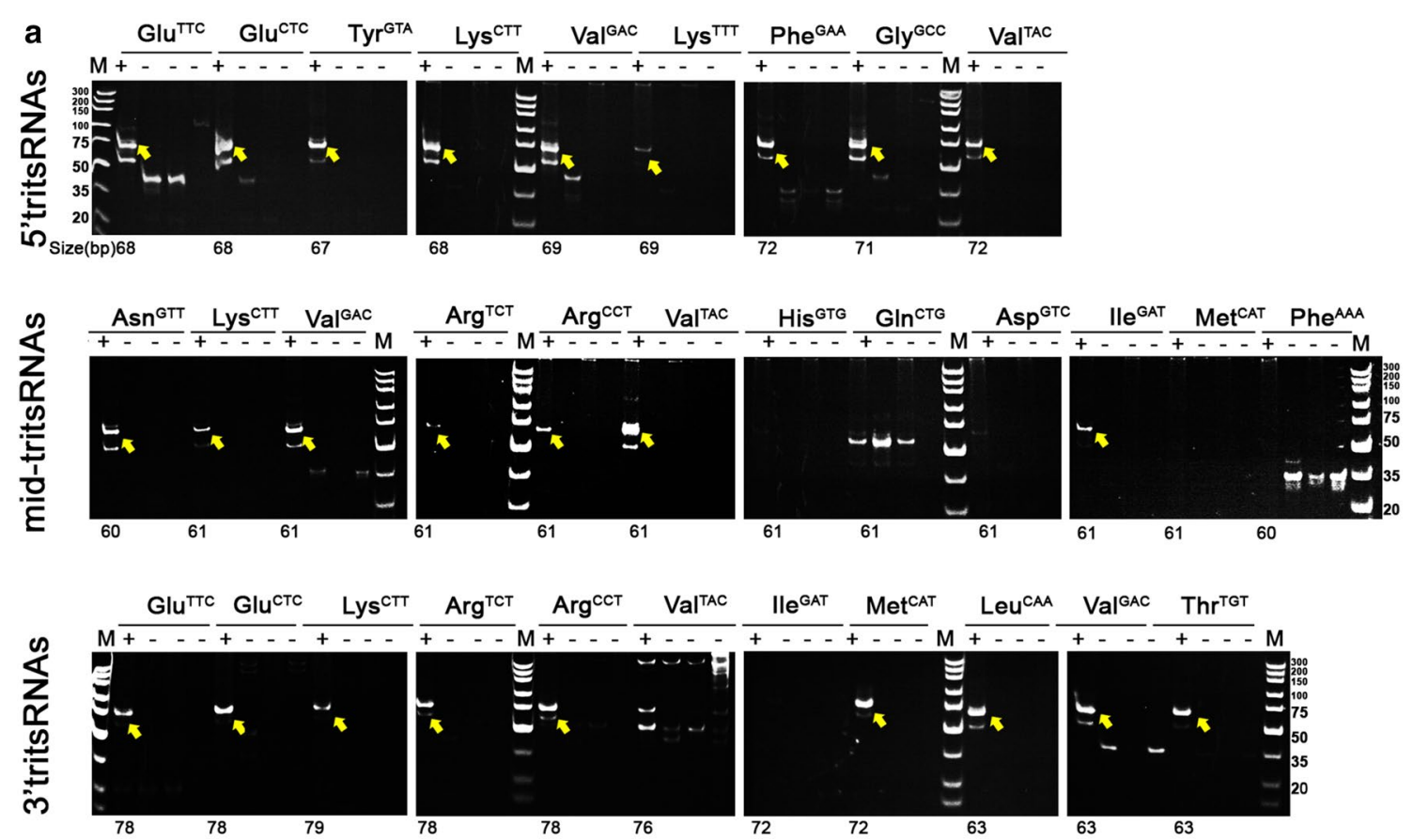

b

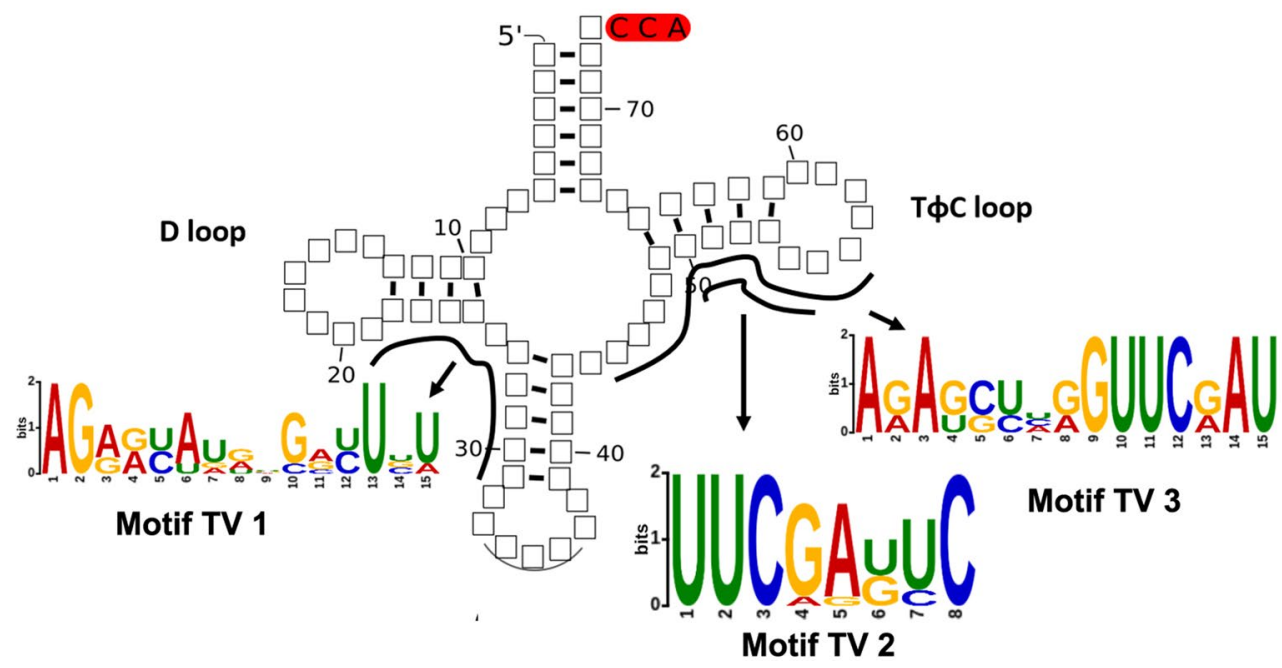

Fig. 4. Experimental confirmation and motif prediction of tritsRNAs. a Reverse transcription (RT)-PCR validation of tritsRNA candidates. Each tritsRNA is shown in four adjacent lanes, which are from left to right: stem-loop RT-PCR with DNase-treated RNA template (left," + "); without RNA template (left middle, "-"); without reverse transcriptase in RT reactions (right middle, "-"); and without template in the PCR reaction as negative control (right, "-"). M indicates the DNA ladder. The yellow arrows refer to the products with the expected size. $\mathbf{b}$ Schematic representation of the motif sequences and locations in the backbone of a mature tRNA.

stress granules [35] in an AGO-independent way. Since the AGO homolog has not been identified in T. vaginalis, it is thus possible that the tsRNA molecules in this parasite might adopt the AGO-independent manner and function in gene regulation.

In T. vaginalis, we identified three categories of tritsRNAs: $5^{\prime}$ tritsRNAs, mid-tritsRNAs and $3^{\prime}$ tritsRNAs. Of interest, both tRFs and tRNA-halves were classified from these tritsRNAs. tRNA-halves are generally believed to be the products of tRNAs under nutritional, biological or physicochemical stresses $[2,4,36]$, while $T$. vaginalis parasites were cultured regularly at low density in our experiment. The presence of tRNA-halves was also noted in a recent study in which nine tRNA-halves were 
discovered from extracellular vesicles of the parasite $T$. vaginalis cultured under conditions of no external stress [7]. It is notable that no extra iron was included in both studies. Iron is essential in the development of T. vaginalis, while iron depletion having the potential to cause the accumulation of nitric oxide in a parasite's cytoplasm, low expression of adhesins on the cell surface, formation of pseudocysts and prolonged survival [3739]. Although the components in Diamond's medium contain iron and could maintain the regular growth of parasites, the ideal level of iron needed remains unclear. Therefore, it is possible that variations in the level of iron in the culture medium place a nutritional stress on the parasite during development. To our knowledge, this is the first time that both tRFs and tRNA-halves have been reported in a protist organism under ordinary culture conditions, and the effect of iron in the generation of tsRNAs need to be further evaluated.

The mechanisms of tsRNAs biogenesis have yet to be revealed because the cleavage loci of known tRNAspecific nucleases still remain to be fully addressed. We therefore analyzed the motifs for tritsRNAs production. Since tRNA structure is highly conserved, there might be other factors involved in the genesis of tritsRNAs. In a tRNA molecule, the D- and T-loops interact with each other to support stabilization. It is thus possible that the motifs might be dependent on the spatial structure of tRNA and were composed of nucleotides that were not adjacent to each other along the sequence. Other unknown mechanisms might also take place in addition to motif recognition in tritsRNAs generation and need to be further elucidated.

\section{Conclusions}

Taken together, this study has provided the first comprehensive evaluation of small non-coding RNAs in T. vaginalis. Previously reported miRNAs were not detected, indicating their absence or that they have little biological function in $T$. vaginalis parasites. Interestingly, three categories of tritsRNAs composed of both classes of tritRFs and tritRNA-halves were identified from $T$. vaginalis under a no stress condition. Future research is needed to understand whether these tsRNAs play a specific role in $T$. vaginalis. Our findings elucidate the profile of small RNAs and provide evidence of tRFs and tRNA-halves in T. vaginalis, which could lead to better understanding of the evolution of RNA processing in the early eukaryotes and the pathogenesis of trichomoniasis.

\section{Supplementary Information}

The online version contains supplementary material available at https://doi. org/10.1186/s13071-020-04570-9.
Additional file 1: Table $\mathbf{S 1}$. Sequences of stem-loop reverse transcription primers. Table S2. Sequences of PCR primers. Table S3. Counts of reads mapped to known miRNA sequences.

Additional file 2: Figure S1. Scatter plots of tritsRNAs between each two categories.

Additional file 3: Figure S2. Size distribution of 5'tritsRNAs from the top 20 highest tsRNAs-expressed tRNA genes in total and unique reads.

Additional file 4: Figure S3. Size distribution of mid-tritsRNAs from the top 20 highest tsRNAs-expressed tRNA genes in total and unique reads.

Additional file 5: Figure S4. Size distribution of 3'tritsRNAs from the top 20 highest tsRNAs-expressed tRNA genes in total and unique reads.

\section{Abbreviations}

AGO: Argonaute protein; cDNA: Complementary DNA; mRNA: Messenger RNA; miRNA: MicroRNA; ncRNA: Non-coding RNA; piRNA: Protein-interacting RNA; PIWI: P-element induced wimpy testis; RPM: Relative number per one million reads; rRNA: Ribosomal RNA; RT-PCR: Quantitative reverse transcription-PCR; snRNA: Small non-coding RNA; snoRNA: Small nucleolar RNA; tRFs: Transfer RNA-derived fragments; tRNA: transfer RNA; tsRNAs: Transfer RNA-derived small RNAs.

\section{Acknowledgements}

The authors are thankful for the technical assistance provided by Aksomics Inc, China.

\section{Authors' contributions}

ZSW, HCZ, CYW, LHZ, ZHW, and XH conceived and designed the experiments. ZSW, ZLW performed the experiments. ZSW, ZLW, JZL analyzed the data.

ZSW, ZLW, HW wrote the manuscript. All authors read and approved the final manuscript.

\section{Funding}

This work has been supported by Major National Science and Technology Program of China for Innovative Drug (2017ZX09101002-001-001), CAMS Innovation Fund for Medical Sciences (CIFMS) (2017-I2M-3-016), NHC Key Laboratory of Echinococcosis Prevention and Control (2020WZK2009), and The Key Laboratory of Vector Biology and Pathogen Control of Zhejiang Province, Huzhou University (Grant No. HUZUL201908).

\section{Availability of data and materials}

The datasets generated and analyzed during the current study are available in the GEO repository under the accession number GSE160464.

\section{Ethics approval and consent to participate}

This work did not require ethics approval.

\section{Consent for publication}

Not applicable.

\section{Competing interests}

The authors declare that they have no competing interests.

\section{Author details}

${ }^{1}$ Department of Microbiology and Parasitology, Institute of Basic Medical Sciences, Chinese Academy of Medical Sciences, School of Basic Medicine, Peking Union Medical College, \#5 Dong Dan San Tiao, Beijing 100005, People's Republic of China. ${ }^{2} \mathrm{NHC}$ Key Laboratory of Systems Biology of Pathogens, Institute of Pathogen Biology, Chinese Academy of Medical Sciences and Peking Union Medical College, Beijing, People's Republic of China. ${ }^{3}$ School of Medicine, Huzhou University and Huzhou Central Hospital, Huzhou 313000, Zhejiang province, China. ${ }^{4}$ Department of Immunology, Guilin Medical University, Guilin 541000, Guangxi province, China. ${ }^{5}$ Blood Chamber, Blood Station of Jinan, Jinan 250000, Shandong province, China. ${ }^{6} \mathrm{NHC}$ Key Laboratory of Echinococcosis Prevention and Control, \#21 linkuo North Road, Chengguan District, Lhasa 850000, Tibet Autonomous Region, People's Republic of China. 
Received: 30 October 2020 Accepted: 28 December 2020

Published online: 29 January 2021

\section{References}

1. Lee RC, Feinbaum RL, Ambros V. The C. elegans heterochronic gene lin-4 encodes small RNAs with antisense complementarity to lin-14. Cell. 1993:75:843-54.

2. Fu H, Feng J, Liu Q, Sun F, Tie Y, Zhu J, et al. Stress induces tRNA cleavage by angiogenin in mammalian cells. FEBS Lett. 2009;583:437-42.

3. Thompson DM, Parker R. The RNase Rny $1 \mathrm{p}$ cleaves tRNAs and promotes cell death during oxidative stress in Saccharomyces cerevisiae. J Cell Biol. 2009;185:43-50.

4. Thompson DM, Parker R. Stressing out over tRNA cleavage. Cell. 2009;138:215-9.

5. Chen Q, Yan M, Cao Z, Li X, Zhang Y, Shi J, et al. Sperm tsRNAs contribute to intergenerational inheritance of an acquired metabolic disorder. Science. 2016;351:397-400

6. Saikia M, Jobava R, Parisien M, Putnam A, Krokowski D, Gao XH, et al. Angiogenin-cleaved tRNA halves interact with cytochrome $c$, protecting cells from apoptosis during osmotic stress. Mol Cell Biol. 2014;34:2450-63.

7. Artuyants A, Campos TL, Rai AK, Johnson PJ, Dauros-Singorenko P, Phillips A, et al. Extracellular vesicles produced by the protozoan parasite Trichomonas vaginalis contain a preferential cargo of tRNA-derived small RNAs. Int J Parasitol. 2020:50:1145-55.

8. Keam SP, Hutvagner G. tRNA-derived fragments (tRFs): emerging new roles for an ancient RNA in the regulation of gene expression. Life (Basel). 2015:5:1638-51.

9. Kumar P, Kuscu C, Dutta A. Biogenesis and function of transfer RNArelated fragments (tRFs). Trends Biochem Sci. 2016;41:679-89.

10. Wang Z, Wei C, Hao X, Deng W, Zhang L, Wang Z, et al. Genome-wide identification and characterization of transfer RNA-derived small RNAs in Plasmodium falciparum. Parasites Vectors. 2019;12:36.

11. Lee YS, Shibata Y, Malhotra A, Dutta A. A novel class of small RNAs: tRNAderived RNA fragments (tRFs). Genes Dev. 2009;23:2639-49.

12. Cai P, Piao X, Hao L, Liu S, Hou N, Wang H, et al. A deep analysis of the small non-coding RNA population in Schistosoma japonicum eggs. PLoS One. 2013;8:e64003.

13. Sobala A, Hutvagner G. Small RNAs derived from the $5^{\prime}$ end of tRNA can inhibit protein translation in human cells. RNA Biol. 2013;10:553-63.

14. Li Z, Ender C, Meister G, Moore PS, Chang Y, John B. Extensive termina and asymmetric processing of small RNAs from rRNAs, snoRNAs, snRNAs, and tRNAs. Nucleic Acids Res. 2012;40:6787-99.

15. Kumar P, Anaya J, Mudunuri SB, Dutta A. Meta-analysis of tRNA derived RNA fragments reveals that they are evolutionarily conserved and associate with AGO proteins to recognize specific RNA targets. BMC Biol. 2014;12:78.

16. Loss-Morais G, Waterhouse PM, Margis R. Description of plant tRNAderived RNA fragments (tRFs) associated with argonaute and identification of their putative targets. Biol Direct. 2013;8:6.

17. Keam SP, Young PE, McCorkindale AL, Dang TH, Clancy JL, Humphreys DT, et al. The human Piwi protein Hiwi2 associates with tRNA-derived piRNAs in somatic cells. Nucleic Acids Res. 2014;42:8984-95.

18. Cole C, Sobala A, Lu C, Thatcher SR, Bowman A, Brown JW, et al. Filtering of deep sequencing data reveals the existence of abundant Dicerdependent small RNAs derived from tRNAs. RNA. 2009;15:2147-60.

19. Liao JY, Guo YH, Zheng LL, Li Y, Xu WL, Zhang YC, et al. Both endosiRNAs and tRNA-derived small RNAs are involved in the differentiation of primitive eukaryote Giardia lamblia. Proc Natl Acad Sci USA. 2014;111:14159-64.

20. Reifur L, Garcia-Silva MR, Poubel SB, Alves LR, Arauco P, Buiar DK, et al. Distinct subcellular localization of tRNA-derived fragments in the infective metacyclic forms of Trypanosoma cruzi. Mem Inst Oswaldo Cruz. 2012:107:816-9.

21. Lambertz U, Oviedo Ovando ME, Vasconcelos EJ, Unrau PJ, Myler PJ, Reiner NE. Small RNAs derived from tRNAs and rRNAs are highly enriched in exosomes from both old and new world Leishmania providing evidence for conserved exosomal RNA packaging. BMC Genomics. 2015;16:151.
22. World Health Organization. Report on global sexually transmitted infection surveillance 2015. Geneva: World Health Organization; 2016.

23. Huang PJ, Lin WC, Chen SC, Lin YH, Sun CH, Lyu PC, et al. Identification of putative miRNAs from the deep-branching unicellular flagellates. Genomics. 2012;99:101-7.

24. Chen XS, Collins LJ, Biggs PJ, Penny D. High throughput genome-wide survey of small RNAs from the parasitic protists Giardia intestinalis and Trichomonas vaginalis. Genome Biol Evol. 2009;1:165-75.

25. Lin WC, Li SC, Lin WC, Shin JW, Hu SN, Yu XM, et al. Identification of microRNA in the protist Trichomonas vaginalis. Genomics. 2009;93:487-93.

26. Diamond LS. The establishment of various trichomonads of animals and man in axenic cultures. J Parasitol. 1957:43:488-90.

27. Chen C, Ridzon DA, Broomer AJ, Zhou Z, Lee DH, Nguyen JT, et al. Realtime quantification of microRNAs by stem-loop RT-PCR. Nucleic Acids Res. 2005;33:e179.

28. Kramer MF. Stem-loop RT-qPCR for miRNAs. Curr Protoc Mol Biol. 2011; Chapter 15:Unit 15.10

29. Ji X, Jiang P, Luo J, Li M, Bai Y, Zhang J, et al. Identification and characterization of miRNAs involved in cold acclimation of zebrafish ZF4 cells. PLoS One. 2020;15:e0226905.

30. Urena-Peralta JR, Alfonso-Loeches S, Cuesta-Diaz CM, Garcia-Garcia F, Guerri C. Deep sequencing and miRNA profiles in alcohol-induced neuroinflammation and the TLR4 response in mice cerebral cortex. Sci Rep. 2018;8:15913.

31. Warf MB, Johnson WE, Bass BL. Improved annotation of C. elegans microRNAs by deep sequencing reveals structures associated with processing by Drosha and Dicer. RNA. 2011;17:563-77.

32. Atayde VD, Tschudi C, Ullu E. The emerging world of small silencing RNAs in protozoan parasites. Trends Parasitol. 2011;27:321-7.

33. Mirande M. Processivity of translation in the eukaryote cell: role of aminoacyl-tRNA synthetases. FEBS Lett. 2010;584:443-7.

34. Gebetsberger J, Wyss L, Mleczko AM, Reuther J, Polacek N. A tRNAderived fragment competes with mRNA for ribosome binding and regulates translation during stress. RNA Biol. 2017;14:1364-73.

35. Emara MM, Ivanov P, Hickman T, Dawra N, Tisdale S, Kedersha N, et al. Angiogenin-induced tRNA-derived stress-induced RNAs promote stressinduced stress granule assembly. J Biol Chem. 2010;285:10959-68.

36. Honda S, Loher P, Shigematsu M, Palazzo JP, Suzuki R, Imoto I, et al. Sex hormone-dependent tRNA halves enhance cell proliferation in breast and prostate cancers. Proc Natl Acad Sci USA. 2015;112:E3816-25.

37. Dias-Lopes G, Saboia-Vahia L, Margotti ET, Fernandes NS, Castro CLF Oliveira FOJ, et al. Morphologic study of the effect of iron on pseudocyst formation in Trichomonas vaginalis and its interaction with human epithelial cells. Mem Inst Oswaldo Cruz. 2017;112:664-73.

38. Cheng WH, Huang KY, Huang PJ, Hsu JH, Fang YK, Chiu CH, et al. Nitric oxide maintains cell survival of Trichomonas vaginalis upon iron depletion. Parasit Vectors. 2015;8:393.

39. Garcia AF, Chang TH, Benchimol M, Klumpp DJ, Lehker MW, Alderete JF. Iron and contact with host cells induce expression of adhesins on surface of Trichomonas vaginalis. Mol Microbiol. 2003;47:1207-24.

\section{Publisher's Note}

Springer Nature remains neutral with regard to jurisdictional claims in published maps and institutional affiliations.

\footnotetext{
Ready to submit your research? Choose BMC and benefit from:

- fast, convenient online submission

- thorough peer review by experienced researchers in your field

- rapid publication on acceptance

- support for research data, including large and complex data types

- gold Open Access which fosters wider collaboration and increased citations

- maximum visibility for your research: over 100M website views per year
}

At BMC, research is always in progress.

Learn more biomedcentral.com/submissions 